\title{
ESTUDO DE CONFIABILIDADE DA APLICAÇÃO DA ESCALA DE FUGL-MEYER NO BRASIL
}

\author{
Maki T ${ }^{1}$, Quagliato EMAB ${ }^{2}$, Cacho EWA ${ }^{3}, \mathrm{PAz}_{\mathrm{LPS}}{ }^{4}$, Nascimento $\mathrm{NH}^{1}$, Inoue MMEA ${ }^{5}$, \\ VIANA MA ${ }^{2}$ \\ ${ }^{1}$ Departamento de Fisioterapia, Universidade de Mogi das Cruzes, Mogi das Cruzes, SP \\ ${ }^{2}$ Setor Distúrbios do Movimento, Departamento de Neurologia, Hospital das Clínicas - Unicamp, Campinas, SP \\ ${ }^{3}$ Fisioterapia Aplicada a Neurologia, Unicamp, Campinas, SP \\ ${ }^{4}$ Fisioterapeuta \\ ${ }^{5}$ Departamento de Fisioterapia, Universidade São Francisco, Bragança Paulista, SP \\ Correspondência para: Tiaki Maki, Rua Padre Vieira, 970 Apto 44, CEP 13015-301, Campinas, SP, \\ e-mail: tiaki_maki@yahoo.com.br
}

Recebido: 04/02/2005 - Aceito: 03/08/2005

\begin{abstract}
RESUMO
Objetivos: Os objetivos do estudo foram realizar uma versão brasileira da escala original de Fugl-Meyer e verificar a confiabilidade da aplicação inter e intra-observador desta versão em pacientes crônicos pós AVC. Método: Participaram do estudo 50 pacientes portadores de hemiparesia, os quais foram submetidos a duas avaliações (confiabilidade intra-observador), realizadas por três fisioterapeutas (confiabilidade interobservador), procedentes de três centros de reabilitação. Resultados: Os resultados demonstraram alta confiabilidade inter e intra-observador da EFM total (IC = 0,99 e 0,98; respectivamente), assim como para todas as subescalas (interobservador IC $=0,99$ a 0,94 ; intra-observador IC $=0,98$ a 0,87 ). Conclusão: Conclui-se neste artigo que não foi verificado conflitos de interpretação na versão brasileira da escala de Fugl-Meyer. Obtivemos alto índice de confiabilidade, tanto intra como interobservador, permitindo assim seu uso como instrumento de avaliação clínica e de pesquisa no Brasil.
\end{abstract}

Palavras-chave: confiabilidade, escala de avaliação de Fugl-Meyer, AVC.

\section{ABSTRACT}

\section{Reliability Study on the Application of the Fugl-Meyer Scale in Brazil}

Objective: The aim of this study was to produce a Brazilian version of the original Fugl-Meyer Assessment Scale and to verify the intrarater and interrater reliability in chronic post-stroke patients. Method: Fifty hemiparetic patients participated in this study. The Fugl-Meyer assessment was applied to them twice (intrarater reliability) by three physiotherapists (interrater reliability), from three rehabilitation centers. Results: The results showed that the whole Fugl-Meyer scale demonstrated high interrater and intrarater reliability (intraclass correlation coefficient $=0.99$ and 0.98 , respectively), and high reliability for each subscale (intraclass interrater $=0.99$ to 0.94 ; intraclass intrarater $=0.98$ to 0.87). Conclusion: It was concluded that the Brazilian Portuguese version of the Fugl-Meyer Assessment Scale did not show any conflicts of interpretation. High intrarater and interrater reliability rates were obtained, thereby allowing this version to be used as instrument for clinical evaluation and research in Brazil.

Key words: reliability, Fugl-Meyer assessment scale, stroke. 


\section{INTRODUÇÃO}

O Acidente Vascular Cerebral (AVC) é um importante problema de saúde pública que se situa entre as quatro principais causas de morte em muitos países, e é responsável por um grande número de pacientes com seqüelas neurológicas ${ }^{1}$.

Nos últimos dezoito anos, várias escalas para avaliar o estado sensório-motor após um AVC têm sido desenvolvidas e usadas ${ }^{2-4}$. Escalas de avaliação funcionais são usadas na prática da reabilitação e em pesquisa para diagnósticos, prognósticos e resposta a tratamentos ${ }^{5}$. Mensurações da recuperação do paciente com seqüela a um AVC, normalmente focalizam a independência nas atividades de vida diária (AVDs), e não são específicas para medir o comprometimento na função sensório-motora ${ }^{6}$.

Uma mensuração do comprometimento motor e sensorial seguido ao AVC, conhecida como Escala de Avaliação de FuglMeyer (EFM), foi desenvolvida e introduzida, em 1975, por Fugl-Meyer et al. ${ }^{7}$. Esta escala foi o primeiro instrumento quantitativo para mensuração sensório-motora da recuperação do $\mathrm{AVC}^{3,8,9}$ e é, provavelmente, a escala mais conhecida e usada para a pesquisa e/ou prática clínica ${ }^{3,4,8}$.

A EFM foi essencialmente desenvolvida com base nos métodos descritos, anteriormente, por Brunnstrom ${ }^{10} \mathrm{e}$ Twitchell ${ }^{11}$, os quais descreveram as seqüências específicas da recuperação motora em pacientes pós-AVC, caracterizando o desempenho e as mudanças no comprometimento motor ${ }^{7,12}$.

As medidas propostas na EFM são baseadas no exame neurológico e na atividade sensório-motora de membros superiores e inferiores, buscando identificar a atividade seletiva e padrões sinérgicos de pacientes que sofreram AVC. Esta escala foi construída seguindo a hipótese que a restauração da função motora nos pacientes hemiplégicos segue um curso definido. Assim, para um paciente com hemiparesia, a volta dos reflexos precede a ação motora voluntária, seguida por completa dependência de sinergias, e o movimento ativo aparecerá sucessivamente menos dependente de reflexos e reações primitivas. Finalmente a completa função motora voluntária com reflexos motores normais pode ser alcançado7.

A EFM é um sistema de pontuação numérica acumulativa que avalia seis aspectos do paciente: a amplitude de movimento, dor, sensibilidade, função motora da extremidade superior e inferior e equilíbrio, além da coordenação e velocidade, totalizando 226 pontos. Uma escala ordinal de três pontos é aplicada em cada item: 0 - não pode ser realizado, 1 - realizado parcialmente e 2 - realizado completamente ${ }^{13}$. Esta escala tem um total de 100 pontos para a função motora normal, em que a pontuação máxima para a extremidade superior é 66 e para a inferior, 34. A avaliação motora inclui mensuração do movimento, coordenação e atividade reflexa de ombro, cotovelo, punho, mão, quadril, joelho e tornozelo ${ }^{3}$. Fugl-Meyer et al. ${ }^{7}$ determinaram uma pontuação de acordo com o nível de comprometimento motor, em que menos que
50 pontos indicam um comprometimento motor severo; 5084 marcante; 85-95 moderado; e 96-99 leve.

Estudos prévios, utilizando a EFM, têm identificado precocemente o comprometimento motor no pós-AVC para um prognóstico a longo prazo da disfunção ${ }^{5}$. Um dos problemas encontrados na clínica, para avaliar o efeito de intervenções durante a reabilitação, é encontrar avaliações válidas, confiáveis e responsivas ${ }^{8}$, tendo em vista que mensurações válidas e confiáveis para medir o estado sensóriomotor são requeridas para decisões clínicas e propósitos de pesquisa ${ }^{14}$.

Os estudos das validações da EFM têm demonstrado, claramente, uma alta confiabilidade intra-observador e interobservadores, tanto em pacientes crônicos (mais de 6 meses), como em pacientes agudos (menos de 6 meses pósAVC) $)^{2,13-15}$.

Tendo em vista que todo instrumento de avaliação deve ser reprodutível através do tempo, ou seja, deve produzir resultados iguais ou muito semelhantes, em duas ou mais administrações para o mesmo paciente, considerando, naturalmente, que seu estado clínico geral não tenha sido alterado $^{16}$, e sabendo-se que uma medição não é objetiva, a não ser que se demonstre que ela possui níveis adequados de confiabilidade ${ }^{17}$, neste estudo, nossos objetivos foram realizar uma versão brasileira da escala original de Fugl-Meyer e verificar a confiabilidade da aplicação inter e intra-observador desta versão.

\section{MATERIAL E MÉTODO}

Participaram do estudo 50 pacientes portadores de AVC crônico (> 12 meses), de ambos os sexos (28 homens e 22 mulheres), com hemiplegia direita (29) e esquerda (21), média de idade de 58 anos (17 - 81), tempo pós-lesional de 53 meses (13 - 127), e procedentes dos centros de reabilitação da Universidade de Mogi das Cruzes, Universidade São Francisco e Universidade Estadual de Campinas - Unicamp.

Foram excluídos do trabalho indivíduos com patologias prévias que pudessem interferir nas avaliações, como por exemplo, doença reumática severa, amputações, alterações ortopédicas ou um AVC prévio, e apresentar déficit cognitivo.

Os pacientes foram esclarecidos previamente sobre os procedimentos que seriam realizados. Após tomarem ciência da pesquisa, os voluntários assinaram um Termo de Consentimento. O estudo foi previamente aprovado pelo Comitê de Ética em Pesquisa da Faculdade de Ciências Médicas da Unicamp (processo No 069/04).

\section{Tradução da EFM}

A tradução para o português da EFM foi realizada com base na versão original de $1975^{7}$. Esse processo contou com dois tradutores bilíngües qualificados e experientes, e posteriormente foi vertida para o inglês por outros dois tradutores de forma independente, enfatizando-se a tradução 
conceitual ${ }^{18-20}$. Uma banca examinadora, composta por um médico neurologista e dois fisioterapeutas, aplicou a versão brasileira da escala em cinco pacientes para verificar se havia entendimento dos itens da avaliação ${ }^{20}$. Após consenso entre estes profissionais, a versão final foi estabelecida. Desenvolveu-se, assim um manual de instruções da escala, detalhando os procedimentos da avaliação e o modelo da escala com sua pontuação (Anexo 1).

\section{TREINAMENTO DOS AVALIADORES}

Antes de coletar os dados da escala para o teste de confiabilidade, três fisioterapeutas foram treinados por um examinador familiarizado com a escala há sete anos. $\mathrm{O}$ treinamento consistiu de aula teórica do manual e treinamento prático com pacientes hemiparéticos pós-AVC, que não fizeram parte do estudo. Durante o treinamento prático, os avaliadores assistiram a um videoteipe do examinador experiente realizando o teste, e os mesmos pontuaram independentemente. As pontuações foram comparadas entre os avaliadores, e as divergências foram discutidas com o treinador. Ainda durante a prática, cada avaliador em treinamento avaliou um paciente e os outros dois pontuaram, independentemente, até que se conseguisse $100 \%$ de concordância entre os examinadores. $\mathrm{O}$ treinamento encerrou-se, quando os examinadores estavam inteiramente familiarizados com a aplicação dos testes e com os procedimentos de pontuação e interpretação.

\section{Verificação da confiabilidade}

Para obter a confiabilidade intra-observador, cada paciente passou por duas avaliações $\left(1^{\mathrm{a}}\right.$ avaliação - teste, $2^{\mathrm{a}}$ avaliação - reteste), com os três avaliadores, em um intervalo de dois dias entre as mensurações, haja vista que o intervalo sugerido entre o teste-reteste é de 1 a 3 dias para a maioria das medidas físicas ${ }^{17}$. Nenhuma mudança relevante no comprometimento sensório-motor desses pacientes crônicos foi esperada entre os intervalos das avaliações. Cada examinador avaliou o mesmo paciente nos dois períodos de avaliação (teste e reteste).

A confiabilidade interobservador foi obtida através de uma avaliação administrada por um dos avaliadores, o qual aplicava o teste, enquanto os outros dois observavam e pontuavam, independentemente, o desempenho do paciente no momento, obtendo-se, assim, três avaliações para cada indivíduo. Os examinadores não conversavam durante os testes, pois o coeficiente de confiabilidade interobservador poderia ser artificialmente inflado ${ }^{17}$.

Coletou-se o tempo de duração da avaliação de cada paciente.

\section{Análise dos dados}

As medidas de concordância foram expressas em termos de coeficientes de correlação e a análise estatística dos dados foi feita utilizando-se o Coeficiente de Correlação Intraclasses (IC). Foi calculada a confiabilidade inter (três avaliadores) e intra-observador para a pontuação total da EFM e para a escala desmembrada em nove subescalas: movimentação passiva, dor, sensibilidade exteroceptiva e proprioceptiva, função e coordenação/velocidade de membro inferior, equilíbrio e função e coordenação/velocidade de membro superior. Foram analisados, para o cálculo da confiabilidade interobservador, os dados dos três avaliadores nos dois momentos da avaliação (teste e reteste). Para verificar o nível de coeficiente de correlação, adotou-se a seguinte pontuação: IC $<0,40-$ concordância fraca, IC $\leq 0,75$ - concordância moderada e IC $>0,75$ alta concordância ${ }^{21}$.

\section{RESULTADOS}

Os coeficientes de correlação da confiabilidade interobservador analisados estão apresentados na Tabela 1. Tanto a confiabilidade interobservador da EFM total, como para todas as subescalas, nos dois momentos das avaliações (teste e reteste), demonstraram alta concordância (IC > 0,75), com IC de 0,99 para a EFM total. Com relação às subescalas, houve uma variação de 0,94 para coordenação/velocidade de membro inferior, a 0,99 para função de membro superior e sensibilidade exteroceptiva.

Observando-se a confiabilidade intra-observador (Tabela 1) da EFM total dos três avaliadores, obteve-se uma forte correlação para todos avaliadores (próximo a um). E, quando se verificaram as subescalas, observou-se, também, uma alta concordância para todos os itens e para os três avaliadores. A função da extremidade superior foi a que obteve os maiores índices de correlação teste-reteste para os três avaliadores (0,98 para todos). E os menores IC foram obtidos para as tarefas de dor $(0,88$, para avaliador 1$)$, coordenação/velocidade de membro inferior $(0,90$, para avaliador 2$)$ e sensibilidade exteroceptiva $(0,87$, para avaliador 3$)$.

\section{DISCUSSÃO}

O presente estudo analisou os dados de 50 pacientes para se obter a confiabilidade interobservador e intraobservador da EFM, além de uma versão em português, já que a maioria dos instrumentos utilizados para a avaliação de pacientes é quase que exclusivamente encontrada na língua inglesa, portanto existe a necessidade de traduzir para a nossa língua, utilizando uma metodologia adequada, assegurando a utilização desta versão por outros profissionais.

A EFM é considerada a mensuração preferida para estudos, pois sua validade já foi estabelecida ${ }^{10}$. A escala obteve aceitação internacional em razão de sua fácil aplicabilidade e apropriada mensuração da recuperação motora na reabilitação. As instruções são relativamente diretas e simples e a avaliação não requer nenhum equipamento especial, em contraste com outras escalas de avaliação $0^{3,13}$. 
Tabela 1. Coeficiente de correlação intraclasse (IC) interobservador para a EFM total e os 9 subitens da primeira (teste) e da segunda avaliação (reteste), e IC intra-observador no teste-reteste para a EFM total e para as 9 subescalas dos três avaliadores.

\section{CONFIABILIDADE INTEROBSERVADOR CONFIABILIDADE INTRA-OBSERVADOR}

\begin{tabular}{lccccc}
\multicolumn{1}{c}{ Tarefas } & Teste & Re-teste & Av. 1 & Av. 2 & Av. 3 \\
\cline { 2 - 6 } & & 0,99 & 0,98 & 0,98 & 0,98 \\
\hline EFM TOTAL & 0,99 & & & \\
SUBESCALAS: & & & & 0,90 \\
Movimentação Passiva & 0,97 & 0,97 & 0,92 & 0,93 & 0,90 \\
Dor & 0,97 & 0,97 & 0,88 & 0,92 & 0,87 \\
Sensibilidade exteroceptiva & 0,98 & 0,99 & 0,89 & 0,92 & 0,93 \\
Sensibilidade proprioceptiva & 0,97 & 0,98 & 0,94 & 0,94 & 0,93 \\
Função de membro inferior & 0,96 & 0,98 & 0,94 & 0,95 & 0,88 \\
Coordenação/velocidade MI & 0,94 & 0,97 & 0,93 & 0,90 & 0,93 \\
Equilíbrio & 0,98 & 0,98 & 0,97 & 0,97 & 0,97 \\
Função de membro superior & 0,99 & 0,99 & 0,98 & 0,98 & 0,98 \\
Coordenação/velocidade MS & 0,95 & 0,97 & 0,94 & 0,95 & 0,90 \\
\hline
\end{tabular}

Com relação à versão final da EFM, a banca examinadora não encontrou conflitos de interpretação durante a execução dos testes, obtendo-se assim uma escala de fácil compreensão. O manual de instruções da escala detalha os procedimentos da avaliação, facilitando assim o aprendizado e uma ampla aplicação desta avaliação por profissionais que trabalham com pacientes pós AVC. Uma limitação do uso da EFM no Brasil está na necessidade de uma norma ou um manual de administração da escala, pois um estudo relata que isto levaria a uma redução de erros durante a utilização deste instrumento ${ }^{14}$, além disso, a aplicação deste deve ser feita por um examinador treinado ${ }^{3}$. O treinamento dos avaliadores não apresentou dificuldades com relação ao entendimento da escala e do manual de instruções, demonstrando ser uma avaliação de fácil aprendizado.

Os resultados demonstraram uma alta confiabilidade interobservador tanto para a EFM total $(\mathrm{IC}=0,99)$, como para todas as subescalas. O menor coeficiente de correlação entre os três observadores nas subescalas ocorreu para as tarefas de coordenação/velocidade de membro inferior e superior (IC=0,94 e 0,95, respectivamente). Esses achados estão de acordo com diversos estudos de confiabilidade interobservadores. Lin et al. ${ }^{15}$ estudaram 176 pacientes pósAVC, e obtiveram um alto coeficiente de correlação para a pontuação total da EFM (IC= 0,93). Duncan et al. ${ }^{2}$ verificaram um alto coeficiente de correlação interobservador em 19 pacientes crônicos para as tarefas de desempenho motor do membro superior e inferior, e concluíram que a EFM é moderadamente confiável em uma população de pacientes pós-AVC. Sanford et al. ${ }^{14}$ também observaram alta confiabilidade para a pontuação total $(\mathrm{IC}=0,96)$ e nas subescalas da EFM (membro superior= 0,97; membro inferior= 0,92; equilíbrio=0,93; e amplitude de movimento $=0,85$ ), com exceção da dor $(\mathrm{IC}=0,61)$, durante os primeiros seis meses de 12 pacientes pós-AVC.

Confrontando os dados dos coeficientes de correlação interobservador da primeira e da segunda avaliação (Tabela 1), observa-se que os IC mantiveram ou aumentaram o valor na segunda avaliação. Esta melhor concordância pode ser atribuída ao fato de que, na segunda avaliação, a experiência pode ter aumentado o desempenho dos avaliadores e os pacientes podem ter sido mais cooperativos ${ }^{22}$.

Uma alta concordância intra-observador foi igualmente encontrada neste estudo, tanto para EFM total para todos os avaliadores, como para todas as subescalas. Os menores coeficientes de correlação foram encontrados para a dor, coordenação/velocidade de membro inferior e sensibilidade exteroceptiva. Esses fatores podem ter sofrido interferência da própria variação natural de um dia para outro, ou a variação da interpretação de um observador com relação ao paciente ${ }^{14}$. Em contrapartida, Lin et al. ${ }^{15}$, em estudo recente, demonstraram com relação à sensibilidade um fraco a moderado índice de correlação (IC de 0,55 a 0,30), concluindo que a mensuração da função sensorial não suporta o uso desta avaliação na clínica de pacientes pós-AVC, sendo necessário estudos futuros para verificar as propriedades psicométricas da EFM. 


\section{Anexo 1}

Escala de Avaliação de Fugl-Meyer em Português

\begin{tabular}{|c|c|}
\hline TESTE & PONTUAÇÃO \\
\hline $\begin{array}{l}\text { I. Movimentação passiva e dor: } \\
\text { - ombro: flexão, abdução 90, rot. ext. e int. } \\
\text { - cotovelo, punho e dedos: flexão e extensão } \\
\text { - } \text { antebraço: pronação e supinação } \\
\text { - quadril: flexão, abdução, rot. ext. e int. } \\
\text { - joelho: flexão e extensão } \\
\text { - tornozelo: dorsiflexão e flexão plantar } \\
\text { - pé: eversão e inversão } \\
\text { Pont. máx: (44 mobilidade) } \\
\quad \text { (44 dor) }\end{array}$ & $\begin{array}{l}\text { Mobilidade: } \\
\text { 0 - apenas alguns graus de movimento } \\
1 \text { - grau de mobilidade passiva diminuída } \\
2 \text { - grau de movimentação passiva normal } \\
\text { Dor: } \\
\text { 0 - dor pronuncia da durante todos os graus de movimento e dor marcante no final da } \\
\text { amplitude } \\
1 \text { - alguma dor } \\
\text { - nenhuma dor }\end{array}$ \\
\hline $\begin{array}{l}\text { II. Sensibilidade: } \\
- \text { Exterocepção: membro superior, palma da } \\
\text { mão, coxa e sola do pé ( ) } \quad \text { Pont. máx: (8) }\end{array}$ & $\begin{array}{l}\mathbf{0} \text { - anestesia } \\
\mathbf{1} \text { - hipoestesia/ disestesia } \\
\mathbf{2} \text { - normal }\end{array}$ \\
\hline $\begin{array}{l}\text { - Propriocepção: ombro, cotovelo, punho, } \\
\text { polegar, quadril, joelho, tornozelo e hálux ( ) } \\
\text { Pont. máx: (16) }\end{array}$ & $\begin{array}{l}\mathbf{0} \text { - nenhuma resposta correta (ausência de sensação) } \\
\mathbf{1} \text { - 3/4 das respostas são corretas, mas há difer ença entre o lado não afeta do } \\
\mathbf{2} \text { - todas as respostas são corretas }\end{array}$ \\
\hline $\begin{array}{l}\text { III. Função motora de membro supe rior } \\
1 \text { - Motricidade reflexa: bíceps/tríceps ( ) (2) }\end{array}$ & $\begin{array}{l}\mathbf{0} \text { - sem atividade reflexa } \\
\mathbf{2} \text { - atividade reflexa presente }\end{array}$ \\
\hline $\begin{array}{l}2 \text { - Sinergia flexora: elevação, retração de } \\
\text { ombro, abdução }+90 \text {, rot. externa, flexão de } \\
\text { cotovelo, supinação ( ) Pont. máx:(12) }\end{array}$ & \begin{tabular}{|l}
$\frac{0-\text { tarefa não pode ser realizada completamente }}{*}$ \\
- tarefa pode ser realizada parcialmente \\
- tarefa é realizada perfeitamente \\
\end{tabular} \\
\hline $\begin{array}{l}3 \text { - Sinergia extensora: adução do ombro, rot. } \\
\text { interna, extensão cot ovelo, pronação Pont:(8) }\end{array}$ & * \\
\hline 4 - Movimentos com e sem siner gia: & a) $*$ \\
\hline $\begin{array}{l}\text { a) mão a coluna } \\
\text { b) flexã o de oml }\end{array}$ & $\begin{array}{l}\text { b) } \mathbf{0} \text { - se o início do mov. o braço é abduzido ou o cotovelo é fletido } \\
\mathbf{1} \text { - se na fase final do mov., o ombro abduz e/ou ocorre flexão de cotovelo } \\
\mathbf{2} \text { - a tarefa é realizada perfeitamente }\end{array}$ \\
\hline c) prono-supinação (cotov. $90^{\circ}$ e ombro $\left.0^{\circ}\right)($ ) & $\begin{array}{l}\text { c) } \mathbf{0} \text { - Não ocorre posiciona/o correto do cotovelo e ombro e/ou pronação e supinação } \\
\text { não pode ser realizada complet/e } \\
\mathbf{1} \text { - prono-supino pode ser realizada com ADM limitada e ao mesmo tempo o ombro } \\
\text { e o cotovelo estejam corr etamente posicionados } \\
\mathbf{2} \text { - a tarefa é realizada completamente }\end{array}$ \\
\hline & $\begin{array}{l}\text { d) } \mathbf{0} \text { - não é tolerado nenhuma flexão de ombro ou desvio da prona ção do antebraço no } \\
\text { INÍCIO do movimento }\end{array}$ \\
\hline pronado ( ) & $\begin{array}{l}\mathbf{1} \text { - realiza parcialmente ou ocorre flexão do cotovelo e o antebraço não se mantêm } \\
\text { pronado na fase TARDIA do movimento } \\
\mathbf{2} \text { - a tarefa pode ser realizada sem desvio }\end{array}$ \\
\hline e) flexão de ombro de $90^{\circ}$ a $180^{\circ}($ ) & $\begin{array}{l}\text { e) } \mathbf{0} \text { - o braço é abduzido e cotovelo fletid o no início do m } \\
\mathbf{1} \text { - o ombro abduz e/ou ocorre flexão de cotovelo na fas } \\
\mathbf{2} \text { - a tarefa é realizada perfeitamente }\end{array}$ \\
\hline $\begin{array}{l}\text { f) prono-supinação (cotov. estendido e ombro } \\
\text { fletido de } 30 \text { a } 90^{\circ} \text { ( ) }\end{array}$ & $\begin{array}{l}\text { f) } \mathbf{0} \text { - Posição não pode ser obtida pelo paciente e/ou prono-supinação não pode ser } \\
\text { realizada perfeitamente } \\
\mathbf{1} \text { - atividade de prono-supinação pode ser realizada mesmo com ADM limitada e ao }\end{array}$ \\
\hline Pont. & $\begin{array}{l}\text { mesmo tempo o ombro e o cotovelo estejam corretamente posicionados } \\
2 \text { - a tarefa é realizada perfeitamente }\end{array}$ \\
\hline $\begin{array}{l}5 \text { - Atividade reflexa normal: ( ) } \\
\text { bíceps / tríceps/ flexor dedos (avalia-se o } \\
\text { reflexo somente se o paciente atingiu nota } 2 \\
\text { para os itens d), e), f) do item anterior) Pont. } \\
\text { máx: (2) }\end{array}$ & $\begin{array}{l}\mathbf{0}-2 \text { ou } 3 \text { reflexos estão hiperativos } \\
\mathbf{1}-1 \text { reflexo esta marcadamente hiperativo ou } 2 \text { estão vivos } \\
\mathbf{2} \text { - não mais que } 1 \text { reflexo esta vivo e nenhum esta hiperativo }\end{array}$ \\
\hline $\begin{array}{l}\text { 6- Controle de punho: } \\
\text { a) Cotovelo } 90^{\circ} \text {, ombro } 0^{\circ} \text { e pronação, c/ } \\
\text { resistência. (assistência, se necessário) ( ) } \\
\text { b) Máxima flexo-extensão de punho, cotov. } 90^{\circ} \text {, } \\
\text { ombro } 0^{\circ} \text {, dedos fletidos e pronação (auxílio se } \\
\text { necessário) ( ) } \\
\text { c) Dorsiflexão com cotovelo a } 0^{\circ} \text {, ombro a } 30^{\circ} \text { e } \\
\text { pronação, com r esistência (auxílio) ( ) } \\
\text { d) Máxima flexo-extensão, com cotov. } 0^{\circ} \text {, } \\
\text { ombro a } 30^{\circ} \text { e pronação (auxílio) ( ) } \\
\text { e) Circundução ( ) } \\
\text { Pont. máx:(10) }\end{array}$ & $\begin{array}{l}\text { a) } \mathbf{0} \text { - o pcte não pode dorsifletir o punho na posição requerida } \\
\mathbf{1} \text { - a dorsiflexão pode ser realizada, mas sem resistência alguma } \\
2 \text { - a posição pode ser mantida contra a lguma resistência } \\
\text { b) } \mathbf{0} \text { - não oc orre mov. voluntário } \\
\mathbf{1} \text { - o pcte não move ativamente o punho em todo grau de movimento } \\
\mathbf{2} \text { - a tarefa pode ser realizada } \\
\text { c) Idem ao a) } \\
\text { d) Idem ao b) }\end{array}$ \\
\hline
\end{tabular}


7 - Mão:

a) flexão em massa dos dedos ( )

b) extensão em massa dos dedos ( )

c) Preensão 1: Art. metacarpofalangeanas (II a V) estendidas e interfalangeanas distal e proximal fletidas. Preensão contra resistência ( )

d) Preensão 2: O paciente é instruído a aduzir o polegar e segurar um papel interposto entre o polegar e o dedo indicador ( )

e) Preensão 3: O paciente opõe a digital do polegar contra a do dedo indicador, com um lápi s interposto ( )

f) Preensão 4: Segurar com firmeza um objeto cilíndrico, com a superfície volar do primeiro e segund o dedos contra os demais ( )

g) Preensão 5: o paciente segura com firmeza uma bola de tênis ( )

Pont. máx: (14)

\section{Coordenação/ Velocidade MS:}

\section{a) Tremor ( )}

b) Dismetria ( )

c) Velocidade: Index-nariz 5 vezes, e o mais rápido que conseguir ( )

Pont. máx: (6)

V. Função motora membro inferior:

Motricid ade Reflexa

A) Aquiles ( )B) Patelar ( ) (4)

1 - Motricid ade reflexa:

Patelar e aquileu / adutor ( ) (2) a)

b) 0 - nenhuma atividade ocorre

1 - ocorre relaxamento (liberação) da flexão em massa

2 - extensão completa (comparado com mão não afetada)

c) $\mathbf{0}$ - posição requerida não pode ser realizada

1 - a preensão é fraca

2 - a preensão pode ser mantida contra considerável resistência

d) 0 - a função não pode ser realizada

1 - o papel pode ser mantid o no lugar, mas não contra um leve puxão

2 - um pedaço de papel é seg urado firmemente contra um puxão

e) $\mathbf{0}$ - a função não pode ser realizada

1 - o lápis pode ser mantido no lugar, mas não contra um leve puxão

2 - o lápis é segurado firmemente

f) $\mathbf{0}$ - a função não pode ser realizada

1 - o objeto interposto pode ser mantido no lugar, mas não contra um leve puxão

2 - o objeto é segurado firmemente contra um puxão

g) $\mathbf{0}$ - a função não pode ser realizada

1 - o objeto pode ser mantido no lugar, mas não contra um leve puxão

2 - o objeto é segurado firmemente contra um puxão

a) $\mathbf{0}$ - tremor marcante/ $\mathbf{1}$ - tremor leve/ $\mathbf{2}$ - sem tremor

b) $\mathbf{0}$ - dismetria marcante/ $\mathbf{1}$ - dismetria leve/ $\mathbf{2}$ - sem dismetria

c) $\mathbf{0}-6$ seg. mais lento que o lado não afetado/ $\mathbf{1}-2$ a 5 seg. mai s lento que o lado não af etado/

2 - menos de 2 segundos de diferença

$\mathbf{0}$ - sem atividade reflexa

2 - atividade reflexa pode ser avaliada

$\mathbf{0}$ - 2 ou 3 reflexos estão marcadamente hiperativos

1 - 1 reflexo esta hiperativ o ou 2 estão vivos

2 - não mais que 1 reflexo esta vivo

2 - Sinergia flexora: flexão quadril, joelho e dorsiflexão (dec.dorsal) ( )

Pont. máx: (6)

3 - Sinergia extensora: exten são de quadril, adução

de quadril, extensão de joelho, flexão plantar ( ) *

Pont max: (8)

\section{4 - Mov. com e sem sinergias:}

a) a partir de leve extensão de joelho, realizar uma

flexão de joelho além de $90^{\circ}$. (sentado) ( )

b) Dorsiflexão de tornozelo (sentado)( )

c) Quadril a $0^{\circ}$, realizar a flexão de joelho mais que $90^{\circ}$ (em pé) ( )

d) Dorsiflexão do tornozelo (em pé) ( )

Pont. máx:(8)

\section{Coordenação./ Velocidade MI:}

a) Tremor ( )

b) Dismetria ( )

c) Velocidade: calcanhar-joelho 5 vez ( ) (dec. Dorsal) Pont. máx: (6)

\section{VII . Equilíbrio}

a) Sentado sem apoio e com os pés suspensos ( )

b) Reação de pára-quedas no lado não afetado ( )

c) Reação de pára-quedas no lado afetado ( )

d) Manter-se em pé com apoio ( )

e) Manter-se em pé sem apoio ( )

f) A poi o único sobre o lado não afetado ( )

g) Apoio único sobre o lado afetado ( )

Pont. máx: (14)

a) $\mathbf{0}$ - sem movimento ativo

$\mathbf{1}$ - o joelho pode ativamente ser fletido até $90^{\circ}$ (palpar os tend ões dos flexores do joelho) 2 - o joelho pode ser fletido além de $90^{\circ}$

b) $*$

c) $\mathbf{0}$ - o joelho não pode ser fletido se o quadril não é fletido simultaneamente

1 - inicia flexão de joelho sem flexão do quadril, porém não atinge os $90^{\circ}$ de flexão de joelho ou flete o quadril durante o término do movimento.

2 - a tarefa é realizada completamente d) $*$

a) $\mathbf{0}$ - tremor marcante/ $\mathbf{1}$ - tremor leve/ $\mathbf{2}$ - sem tremor

b) $\mathbf{0}$ - dismetria marcante/ $\mathbf{1}$ - dismetria leve/ $\mathbf{2}$ - sem dismetria

c) $\mathbf{0}-6$ seg. mais lento que o lado não afetado/ $\mathbf{1}-2$ a 5 seg. mai s lento que o lado afetado/ 2 - menos de 2 seg und os de diferença

a) $\mathbf{0}$ - não consegue se manter sentado sem apoio/ $\mathbf{1}$ - permanece sentado sem apoi o por pouco tempo/ $\mathbf{2}$ - permanece sentad o sem apoio por pelo menos 5 min. e regula a postura do corpo em relação a gravidade

b) $\mathbf{0}$ - não oc orre abdução de ombro, extensão de cotovelo para evitar a queda/ $\mathbf{1}$ - reação de pára-quedas parcial/ $\mathbf{2}$ - reação de pára-quedas normal

c) idemaob)

d) $\mathbf{0}$ - não consegue ficar de pé/ $\mathbf{1}$ - de pé com ap oio máximo de outros/ $\mathbf{2}$ - de pé com apoio mínimo por 1 min

e) $\mathbf{0}$ - não consegue ficar de pé sem apoio/ $\mathbf{1}$ - pode permanecer em pé por 1 min e sem oscilação, ou por mais tempo, porém com alguma oscilação/ 2 - bo m equilíbrio, pode manter o equilíbrio por mais que 1 minuto com segurança

f) $\mathbf{0}$ - a posição não pode ser mantida por mais que 1-2 seg (o scilação)/ $\mathbf{1}$ - consegue permanecer empé, com equilíbrio, por 4 a 9 segundos/ $\mathbf{2}$ - pode manter o equilíbrio nesta posição por mais que 10 segund os

g) $\mathbf{0}$ - a posição não pode ser mantida por mais que 1-2 segundos (oscilação)

1 - consegue permanecer em pé, com equilíbrio, por 4 a 9 segundos

$\mathbf{2}$ - pode manter o equilíbrio nesta posição por mais que 10 segund os 
A EFM é uma eficiente avaliação que pode ser realizada em, aproximadamente, 30 minutos, segundo Duncan et al. ${ }^{2}$. Nesse estudo, o tempo médio de administração da escala foi 40 minutos para avaliadores já treinados.

\section{CONCLUSÃO}

Conclui-se que a versão brasileira da EFM não apresentou conflitos de interpretação, além de ser uma escala de fácil aprendizado e aplicabilidade.

Obteve-se alto índice de confiabilidade, tanto intraobservador como interobservador, garantindo a replicabilidade desta versão brasileira da EFM, permitindo assim seu uso como instrumento de avaliação clínica e de pesquisa em nosso meio.

Agradecimentos: Nossos agradecimentos vão, em especial, aos profissionais dos centros de reabilitação da Universidade de Mogi das Cruzes: Camila C. Reys, Érika M. Siqueira e Vanessa A. Faria e da Universidade São Francisco: Daniele M. Senoni, Camila A. Oliveira, Michelle C. de Moraes, Marcela S. Miranda, Luciana V. Colucci, Andreia V. Moreira, Vanessa O. Silva, Flávia F. Bueno e Caroline C. Muner, e aos pacientes que tornaram possível esta pesquisa.

\section{REFERÊNCIAS BIBLIOGRÁFICAS}

1. Rowland LP. Merrit Tratado de neurologia. $9^{\mathrm{a}}$ ed. Rio de Janeiro: Ed. Guanabara Koogan; 1997. p. 177-230.

2. Duncan PW, Prost M, Nelson SG. Reliability of the Fugl-Meyer Assessment of sensoriomotor recovery following cerebrovascular accident. Phys Ther 1983; 63: 1606-1610.

3. Gladstone DJ, Daniells CJ, Black SE. The Fugl-Meyer Assessment of motor recovery after stroke: a critical review of its measurement properties. Neurorehabil Neural Repair 2002; 16: 232-240.

4. Malouin F, Pichard L, Bonneau C, Durand A, Corriveau D. Evaluating motor recovery early after stroke: comparison of the Fugl-Meyer assessment and the motor assessment scale. Arch Phys Med Rehabil 1994; 75: 1206-1212.

5. Jansa J, Pogacnik T, Gompertz PN. An evaluation of the extended Barthel Index with acute ischemic stroke patients. Neurorehabil Neural Repair 2004; 18: 37-41.

6. Nakayama H, Jorgensen HS, Raaschou HO, Olsen TS. Compensation in recovery of upper extremity function after stroke: The Copenhagen stroke study. Arch Phys Med Rehabil 1994; 75: 852-857.
7. Fugl-Meyer AR, Jaasko L, Leyman I, Olsson S, Steglind S. The post-stroke hemiplegic patient: 1 . A method for evaluation of physical performance. Scand J Rehab Med 1975; 7: 13-31.

8. Van der Lee JH, Beckerman H, Lankhorst GJ, Bouter LM. The responsiveness of the Action Research Arm Test and the FuglMeyer assessment scale in chronic stroke patients. J Rehab Med 2001; 33: 110-113.

9. Duncan PW, Goldsteins LB, Horner RD, Landsman PB, Samsa GP, Matchar DB. Similar motor recovery of upper and lower extremities after stroke. Stroke 1994; 25: 1181-1188.

10. Brunnstrom S. Motor Testing Procedures in hemiplegia. J Am Phys Ther Ass 1966; 46: 357-375.

11. Twitchell TE. The restoration of motor function following hemiplegia in man. Brain 1951; 74: 443-480.

12. Gowland C. Standardized physical therapy measurements for assessing impairment and disability following stroke. Neurol Report 1991; 15: 9-11.

13. Fugl-Meyer AR. Post stroke hemiplegia: Assessment of physical properties.Scand J Rehab Med 1980; suppl 7: 85-93.

14. Sanford J, Moreland J, Swanson LR, Stratford PW, Gowland C. Reliability of the Fugl-Meyer assessment for testing motor performance in patients following stroke. Phys Ther 1993; 73: 447-454.

15. Lin JH, Hsueh P, Sheu CF, Hsieh CL. Psychometric properties of the sensory scale of the Fugl-Meyer Assessment in stroke patients (Abstr). Clin Rehabil 2004; 8: 391-397.

16. Ciconelli RM, Ferraz MB, Santos W, Meinão I, Quaresma MR. Tradução para a língua portuguesa e validação do questionário genérico de avaliação de qualidade de vida SF-36 (Brasil SF-36). Rev Bras Reumatol 1999; 39: 143-150.

17. DeLisa JA. Tratado de medicina de reabilitação. $3^{\mathrm{a}}$ ed. São Paulo: Ed. Manole; 2002. P. 115- 144.

18. Fleck MPA, Leal OF, Louzada S et al. Desenvolvimento da versão em português do instrumento de avaliação de qualidade de vida da OMS (WHOWOL -100). Rev Bras Psiquiatr 1999; 21: 19-28.

19. Falcão DM, Ciconelli RM, Ferraz MB. Translation and cultural adaptation of quality of life questionnaires: Na evaluation of methodology. J Rheumatol 2003; 30: 379-85.

20. Guillemin F, Bombardier C, Beaton D. Cross-cultural adaptation of health-related quality of life measures: literature review and proposed guidelines. J Clin Epidemiol 1993; 46(12) 141732.

21. Fleiss, J.L. Statistical Methods for rates and proportions. $2^{\text {nd }}$ ed. New York: John Wiley \& Sons; 1999. p. 252.

22. Wood-Dalphinee SL, Williams I, Shapiro SH. Examining outcome measures in a clinical study of stroke. Stroke 1990; 21: 731739. 